\title{
CYTOLETHAL DISTENDING TOXIN A, B AND C SUBUNIT PROTEINS ARE NECESSARY FOR THE GENOTOXIC EFFECT OF ESCHERICHIA COLI CDT-V
}

\author{
Frederic TAIEB ${ }^{1}$, Domonkos SvÁB ${ }^{2}$, Claude WATRIN ${ }^{1}$, Eric OSWALD ${ }^{1}$ and István TóTH ${ }^{2 *}$ \\ ${ }^{1}$ USC INRA 1360-CPTP, U1043 INSERM, Pathogénie moléculaire et cellulaire des \\ infections à Escherichia coli, CHU Purpan, Toulouse, France; ${ }^{2}$ Enteric Bacteriology and \\ Foodborne Zoonoses Group, Institute for Veterinary Medical Research, Centre for \\ Agriculture Research, Hungarian Academy of Sciences, Hungária krt. 21, \\ H-1143 Budapest, Hungary
}

(Received 4 June 2014; accepted 29 October 2014)

Cytolethal distending toxins (CDT) are considered the prototype of inhibitory cyclomodulins, and are produced by a wide range of Gram-negative pathogenic bacteria, including Escherichia coli strains of various sero- and pathotypes. $\mathrm{CDT}$ is a heterotripartite toxin consisting of three protein subunits, $\mathrm{CdtA}, \mathrm{CdtB}$ and $\mathrm{CdtC}$. The active subunit, CdtB has DNase activity and causes DNA damage and cell cycle arrest in the target cell. However, several studies have highlighted different roles for CdtA and $\mathrm{CdtC}$ subunits. In order to reveal the necessity of CdtA and $\mathrm{CdtC}$ subunit proteins in the CDT-specific phenotype, expression clones containing the $c d t-V$ subunit genes were constructed. Using cell culture assays, we demonstrated that clones expressing only the $\mathrm{CdtB}$ subunit or in combination with only $\mathrm{CdtA}$ or $\mathrm{CdtC}$ were unable to trigger the specific cell cycle arrest and changes in cell morphology in HeLa cells. At the same time, the recombinant clone harbouring the whole $c d t-V$ operon caused all the CDT-associated characteristic phenotypes. All these results verify that all the three CDT subunit proteins are necessary for the genotoxic effect caused by CDT-V.

Key words: Cytolethal distending toxin, CDT-V, E. coli, genotoxic effect

Cytolethal distending toxins (CDT) establish a family of toxins with emerging importance in pathogenic Escherichia coli and numerous other Gramnegative pathogenic bacteria (reviewed by Gargi et al., 2012) and are considered the prototypic family of inhibitory cyclomodulins (Nougayrède et al., 2005; Oswald et al., 2005).

Five types of CDT (CDT-I to CDT-V) have been reported in pathogenic E. coli strains (Tóth et al., 2009a); among them CDT-V is the most recently identified (Janka et al., 2003), and the only type to be detected in Shiga toxin

"Corresponding author; E-mail: tothi@vmri.hu; Phone: 0036 (1) 252-2455; Fax: 0036 (1) 252-1069 
producing (STEC) and enterohaemorrhagic (EHEC) E. coli (Orth et al., 2006; Hinenoya et al., 2009; Hinenoya et al., 2014). Additionally, CDT-V has been detected in several strains of uncertain pathotype (Hinenoya et al., 2009; Tóth et al., $2009 \mathrm{~b}$ ), and in some of these strains it is the only identified toxin so far (Tóth et al., 2009b).

Most CDTs form a heterotripartite complex of three subunits, CdtA, CdtB, and CdtC (Gargi et al., 2012). Among them CdtB has DNase activity (Elwell and Dreyfus, 2000), causing DNA damage in the target cell's nucleus, which, through the DNA damage response (DDR) mechanisms, ultimately causes cell cycle arrest between the G2/M phase and the characteristic transformation of the intoxicated cells into giant, elongated mononuclear cells (Comayras et al., 1997; Pérès et al., 1997; Bielaszewska et al., 2005). In the case of cells with fibroblast origin, the cell cycle arrest occurs in the G1/S phase (Belibasakis et al., 2004).

The proposed role of CdtA and $\mathrm{CdtC}$ is the transport of $\mathrm{CdtB}$ to the target cell and the attachment of the toxin to the cell surface, and it has been verified in the case of Campylobacter jejuni (Lara-Tejero and Galán, 2001) and Aggregatibacter actinomycetemcomitans (Nesić et al., 2004; Yamada et al., 2006). However, there is increasing evidence suggesting that despite the similar genotoxic activity, there are differences between the molecular mechanisms used by the different CDT types in interaction with the host cell (McSweeney and Dreyfus, 2005; Eshraghi et al., 2010; Gargi et al., 2013). The exact role of the CdtA and $\mathrm{CdtC}$ subunits is not well understood in the case of E. coli CDT, and no data are available regarding their roles in CDT type $\mathrm{V}$.

The aim of the present study was to assess the roles of the individual CDT-V subunits by cloning the $c d t-V$ genes into an expression vector in different combinations, and evaluating the CDT-specific effect of the clones on tissue cultures.

\section{Materials and methods}

Bacterial strains and human cell cultures. The atypical (stx- and eae-) E. coli O157:H43 strain T22 carrying the $c d t-V$ operon (Tóth et al., 2009b) was used as donor of $c d t$ genes. The E. coli $\mathrm{K}-12$ strain BL21(DE3) was used as recipient for the cloned constructs. HeLa (ATCC CCL-2) cells were maintained by serial passage in Dulbecco's Modified Eagle medium (DMEM GlutaMAX ${ }^{\mathrm{TM}}$, Gibco), supplemented with $10 \%$ fetal calf serum (FCS), non-essential amino acids (Gibco) and $50 \mu \mathrm{g} \mathrm{ml}^{-1}$ gentamicin at $37{ }^{\circ} \mathrm{C}$ in a $5 \% \mathrm{CO}_{2}$ atmosphere.

Construction of clones carrying cdt subunit genes. Recombinants were constructed using PCR-amplified CDT subunit genes in combinations of $A B C$, $A B, B C$ and $B$ from the $c d t-V$ genes of $E$. coli $\mathrm{O} 157: \mathrm{H} 43$ strain T22. The primers used for amplification were designed to match the very first and last bases of the start and stop codons of the respective genes, carried cleavage sites of EcoRI or 
Xhol, and three timins on the 5' end to facilitate annelation, based on the sequence of the $c d t-V$ operon of the strain determined in an earlier study (Sváb et al., 2013; Table 1). All reactions were performed using Platinum Taq HiFi (Sigma-Aldrich, St. Louis, MO, USA), with an annealing temperature of $42{ }^{\circ} \mathrm{C}$. PCR fragments were purified with GenElute PCR Clean-up Kit (Sigma-Aldrich, St. Louis, MO, USA). The proper PCR fragments as well as the pET28b expression vector were digested with the appropriate endonucleases and were ligated by T4 ligase (Fermentas, Vilnius, Lithuania) for $1 \mathrm{~h}$ at room temperature. Competent cells of the recipient bacterial strain were transformed by electroporation. Transformants were selected by growing on lysogeny broth (LB) agar plates supplemented with $25 \mu \mathrm{g} \mathrm{ml}^{-1}$ kanamycin as a selective agent. Resistant clones were tested by PCR and plasmid analysis.

Table 1

Primers used for amplifying $c d t-V$ genes for cloning

\begin{tabular}{lcl}
\hline Primer name & Target gene & \multicolumn{1}{c}{ Sequence $5{ }^{\prime}->3^{\prime}$} \\
\hline CDTAfwEcorI & $c d t A$ & TTTGAATTCAATGGCTAATAAATACACACC \\
cdtA_rev_xhoi & $c d t A$ & TTTCTCGAGTTATTACAGTCCTGTTTATGA \\
cdtbfw_ecori & $c d t B$ & TTTEATTCATGAAAAAATATATTATATC \\
cdtbrev_xhoi & $c d t B$ & TTTETCGAGTTATCGTCTGGAAACGCCAA \\
cdtcfw_ecori & $c d t C$ & TTTEATTCATGAAGAGATTAATAATTAT \\
cdtcrev_xhoi & $c d t C$ & TTTETCGAGTTAAATAATAGGCGATTCAG \\
\hline
\end{tabular}

All primers were designed for this study based on GenBank sequence no. KC618326.1 (Sváb et al., 2013). The restriction sites of EcoRI and XhoI are underlined. All PCRs were run with an annealing temperature of $42{ }^{\circ} \mathrm{C}$, as described in the Materials and methods section

Biomass measurement assays. Ten $\mathrm{ml}$ of LB culture of BL21DE3 expressing the different combinations of CDT subunits were incubated at $37^{\circ} \mathrm{C}$ with vigorous shaking until the optical density $(\mathrm{OD})$ reached 0.5 to 0.6 before $3-\mathrm{h}$ induction with $0.5 \mathrm{mM}$ of IPTG. Supernatant was $0.22 \mu \mathrm{m}$ filtered and was concentrated to $250 \mu \mathrm{l}$ using a centrifugal concentrator (10 kDa cut-off, Vivaspin). Concentrated supernatant was serially diluted 2 times in a 96-well microplate and $100 \mu \mathrm{l}$ of diluted sample were added to HeLa cells cultivated in a 96-well culture microplate $(30,000 \mathrm{HeLa}$ cells per well) in $100 \mu \mathrm{l}$ of DMEM supplemented with $10 \%$ FCS. Quantification of biomass was performed as previously described (De Rycke et al., 1996). After 3 days of culture, cells were fixed with $3.7 \%$ formaldehyde in PBS and then stained with a $1 \%(\mathrm{w} / \mathrm{v})$ solution of methylene blue in borate buffer for $10 \mathrm{~min}$. After five washes in borate buffer, methylene blue was extracted with $0.1 \mathrm{M} \mathrm{HCl}$ and the OD of the staining solution was read at $660 \mathrm{~nm}$ in a microplate reader. Alternatively, cells were stained with Giemsa stain solution (Sigma) for microscopic examination according to the manufacturer's protocol after 3 days of culture. 
TAIEB et al.

Flow cytometry assays. Cell cycle analysis was performed as previously described (Taieb et al., 2006). Six hundred thousand HeLa cells were treated with 1:10 dilution of concentrated supernatant or with purified CDT-I for $24 \mathrm{~h}$ (Taieb et al., 2006). Cells were then trypsinised, resuspended in PBS and fixed in $70 \%$ ethanol overnight at $-20{ }^{\circ} \mathrm{C}$. Cells were washed and resuspended in PBS containing $15 \mathrm{mg} \mathrm{ml}^{-1}$ propidium iodide and $100 \mathrm{mg} \mathrm{ml}^{-1}$ RNase A. Flow cytometry analyses were performed on a FACScalibur flow cytometer (Becton Dickinson). Data were analysed using FlowJo software (Tree Star). Percentage of G1, S and G2 populations were calculated using the Watson-pragmatic and the Dean-Jett-Fox models.

Western blot analysis. Experiments were performed as described previously (Taieb et al., 2006). For western blot analysis of total proteins, 4-8 $\times 10^{5}$ HeLa cells exposed to supernatants of study strains were lysed in $80 \mu 1$ of $1 \times$ Laemmli loading buffer, sonicated for $5 \mathrm{~s}$ to shear DNA, then boiled for $5 \mathrm{~min}$. Proteins were separated on 4-12\% NuPage gradient gels (Invitrogen) and transferred to PVDF membranes. The membranes were blocked in $10 \mathrm{mM}$ Tris $\mathrm{pH}$ $7.8,150 \mathrm{mM} \mathrm{NaCl}, 0.1 \%$ Tween 20 (TBST), $10 \%$ non-fat dry milk, then probed with primary antibody $(0.5 \mu \mathrm{g} / \mathrm{ml})$ in TBST, $5 \%$ non-fat dry milk. The primary antibodies were anti-phosphorylated $\mathrm{H} 2 \mathrm{AX}$ (pH2AX) (Upstate), antiphosphorylated CDK1 (pY15, Cell Signalling Technology), and anti-actin (ICN) antibodies. Bound antibodies were visualised with horseradish peroxidaseconjugated secondary antibody. Acquisitions were performed with a Molecular Imager Chemi Doc XRS System (Bio-Rad).

\section{Results}

HeLa cell biomass assays. In order to investigate the function of CDT-V subunit proteins, we inserted the genes encoding $\mathrm{CdtA}, \mathrm{CdtB}$ and $\mathrm{CdtC}$ proteins into $\mathrm{pET} 28 \mathrm{~b}$ expression vectors and constructed clones of the $E$. coli $\mathrm{K}-12$ strain BL21(DE3). The genes were cloned in the following combinations: $c d t-V A B C$, $c d t-V A B, c d t-V B C$ and $c d t-V B$.

Hela cells were exposed to serial dilutions of the concentrated supernatants of the clones. The greatest decrease of biomass could be observed in cultures exposed to the supernatant of the $c d t-V A B C$ clone, followed by the cultures treated with supernatants of the $c d t-V A B$ and $c d t-V B C$ clones, respectively. In the two latter cases, decrease in biomass could also be observed at higher dilutions, when compared to the controls and to the cells treated with the supernatant of the $c d t-V B$ clone (Fig. 1A), albeit to a considerably lesser extent than in the case of $c d t-V A B C$. Morphological changes were monitored by light microscopy, and cytopathic effect was evident with cell distension for HeLa cells intoxicated only with the supernatant fraction of the culture expressing the three subunits, 
whereas other combinations of CDT-V subunits did not exhibit such distending activity (Fig. 1B).

CDT-specific effects of clones on the cell cycle of HeLa cells. To further explore the mode of action of CDT-V subunit proteins, HeLa cells were incubated in the presence of the culture supernatant of BL21(DE3) E. coli cells containing pET28 vector expressing the four gene combinations described. Purified standard amount of CDT-I was used as positive control (Taieb et al., 2006). Twenty-four $\mathrm{h}$ after treatment, cell cycle distribution was analysed by flow cytometry. Our results showed that the clone with the $c d t-V A B C$ expressed a functional CDT that induced accumulation of cells in the G2-phase or mitosis (Mphase) with a 4 n DNA content (37\% versus $12 \%$ in control cells), albeit with a reduced titre when compared to the purified CDT protein used as control (Fig. $2 \mathrm{~A}$ ). The other constructs expressing a combination of two CDT subunits (AB or $\mathrm{BC}$ ) or B subunit alone did not cause cell cycle arrest (with a population of cells in $\mathrm{G} 2$ and $\mathrm{M}$ phases ranging from $15 \%$ to $16 \%$ ) as compared to the control culture.

To further characterise the CDT-V induced cell cycle arrest, we examined the phosphorylation status of the eukaryotic inducer of the G2 to M phases transition, the CDK1-cyclin B complex. Western blot analysis showed that purified CDT-I as well as CDT-V ABC prevented entry in mitosis by maintaining CDK1 in its inhibitory phosphorylated form, indicating that cells are arrested in the G2phase (Fig. 2B). Moreover, we observed phosphorylation of histone H2AX, a sensitive marker of DNA double strand breaks (DSBs), only in cultures exposed to the supernatant of the clone expressing $c d t-V A B C$ (Fig. 2B). Taken together, these results show that CDT-V induces DSBs that result in activation of the DNA damage response, which eventually leads to inhibition of the mitosis inducer, CDK1 and subsequent accumulation of cells in the G2 phase.

\section{Discussion}

In the case of CDT-V produced by E. coli, the roles of individual CDT protein subunits in the cytotoxic effect were unknown. Using clones expressing the subunit genes of $E$. coli CDT-V in different combinations, we have found that, besides the active $\mathrm{B}$ subunit, the $\mathrm{A}$ and $\mathrm{C}$ subunits are both necessary to induce the characteristic cell cycle arrest and distended cell morphology, as the clones lacking any of the CDT-V subunit proteins were unable to cause these specific effects. As expected, we showed that CDT activity was related to its described DNase activity as treated cells exhibited DNA double strand breaks. We also revealed that CDT-V-associated double strand breaks activated the DNA damage response, leading to CDK1 inhibitory phosphorylation and subsequent cell cycle arrest. 
A

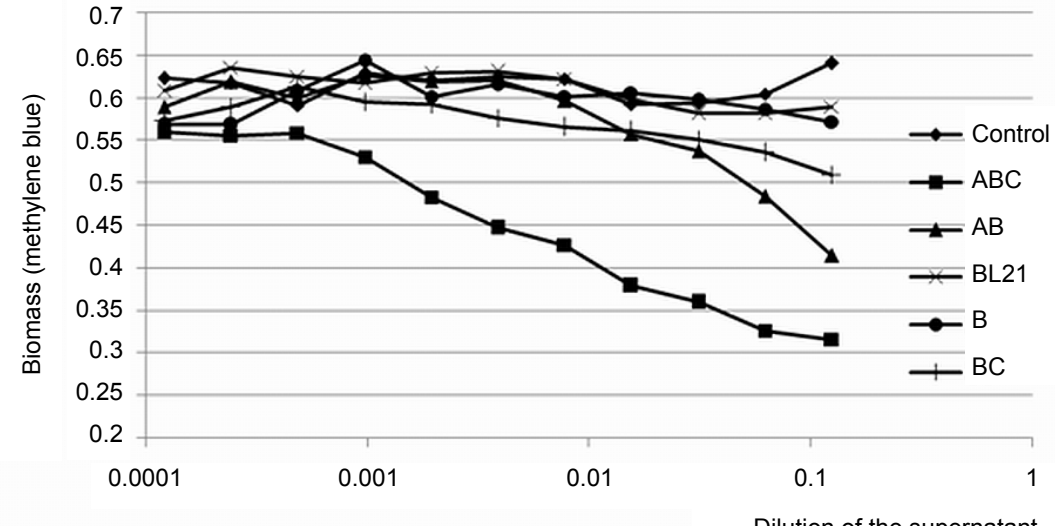

B

CDT-V ABC

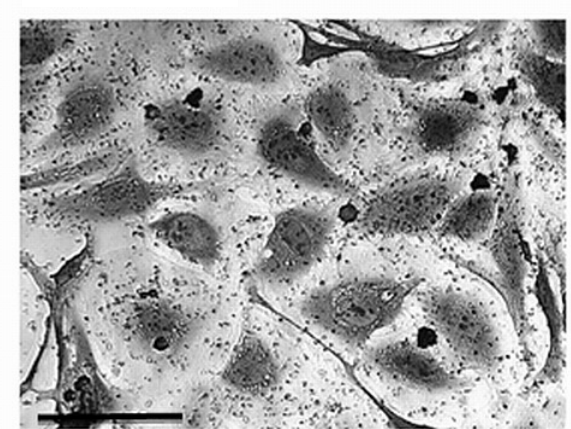

CDT-V B

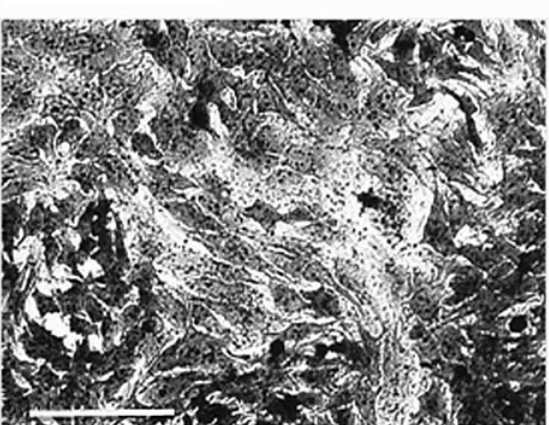

Non-treated

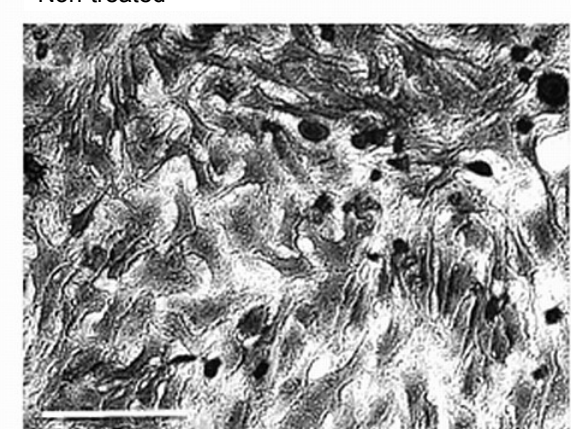

Fig. 1. Cytopathic effect of CDT-V ABC in HeLa cells. (A) Biomass of HeLa cells after 3-day exposure to serial dilutions of the supernatant of BL21(DE3) expressing the indicated CDT subunits. Biomass was expressed as absorbance of methylene blue. (B) HeLa cells exposed as in (A) with supernatant of the indicated clones and stained with Giemsa. Bars represent $100 \mu \mathrm{m}$. Characteristic distended cells are visible when treated with the supernatant containing CDT-V ABC 
A

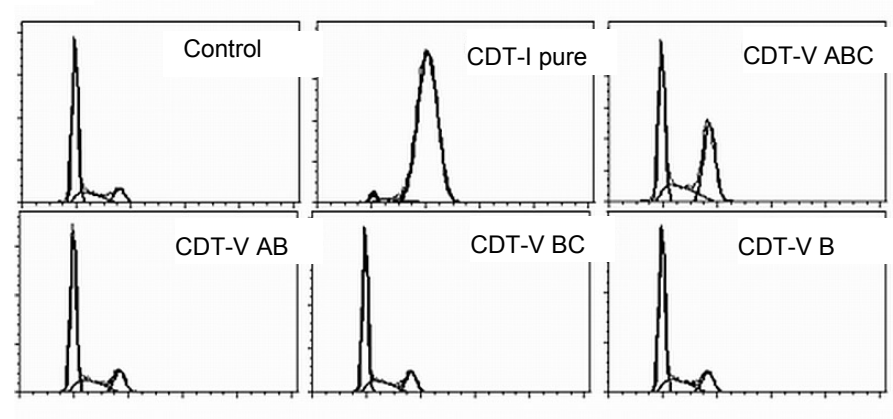

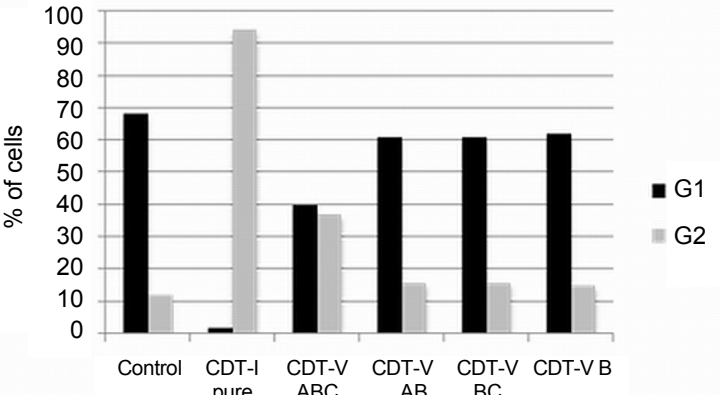

$\begin{array}{ccc}\text { CDT-I } & \text { CDT-V } \\ \text { pure } & A B C\end{array}$

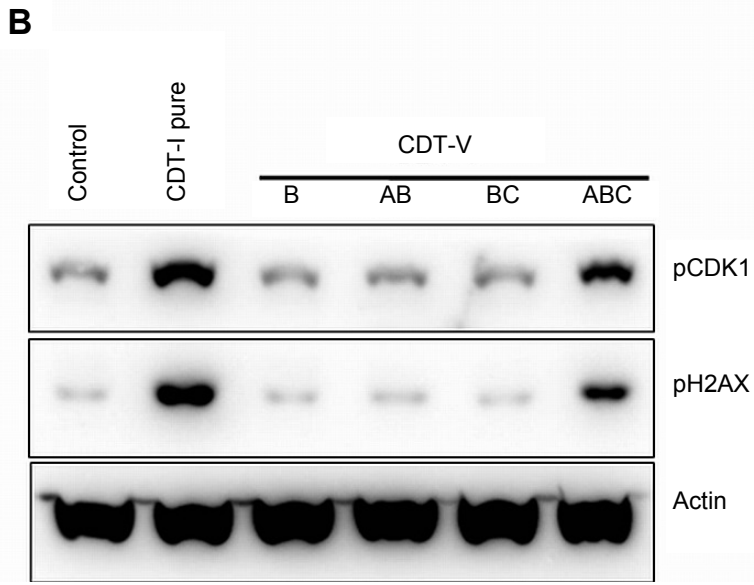

Fig. 2. CDT induces cell cycle arrest and DNA double strand breaks. HeLa cells were treated with supernatant of BL21(DE3) (10 $0^{-1}$ dilution) expressing the indicated CDT subunits for $24 \mathrm{~h}$ and analysed for DNA content by flow cytometry following propidium staining. Percentages of cells in G2/M were calculated using Dean-Jett-Fox model and reported in the right panel. HeLa cells were treated as in (A) and protein extracts were probed with anti-phosphorylated $\mathrm{H} 2 \mathrm{AX}(\mathrm{pH} 2 \mathrm{AX})$, anti-phosphorylated CDK1 (pCDK1) and anti-actin antibodies 
Although the heterotripartite model of the toxin has been confirmed in multiple cases (Nougayrède et al., 2005; Oswald et al., 2005; Tóth et al., 2009a; Gargi et al., 2012), there have been cases where the authors reported characteristic CDT activity in the case of CDTs missing either the A or C subunit, as with the CDT produced by Aggregatibacter actinomycetemcomitans (De Rycke et al., 1996; Taieb et al., 2006). The Aggregatibacter CDT was found to be functional, albeit with a reduced titre, when lacking the A (Akifusa et al., 2001) or the C subunit (Saiki et al., 2001). These findings were later explained by the work of Damek-Poprawa et al. (2012) who reported that after the internalisation of CdtB, CdtA remains on the cell surface, while $\mathrm{CdtC}$ migrates into the cytosol, suggesting that it acts as a chaperone to CdtB. In the present study, the slight decrease in cell mass observable in HeLa cultures after 3 days of exposure to supernatants containing $\mathrm{CdtB}+\mathrm{CdtC}$ combination, and more noticeably in the case of $\mathrm{CdtB}+$ $\mathrm{CdtA}$, might suggest similar mechanisms.

The CDT produced by Haemophilus ducreyi was found to exhibit a slight activity when lacking the A subunit (Deng et al., 2001), although the molecular background of this discrepancy has not been elaborated yet. In the case of this CDT type, when compared to the CDT produced by E. coli, Gargi et al. (2013) found that while endosome acidification inhibits the transport of $H$. ducreyi $\mathrm{CdtB}$ to the nucleus, E. coli $\mathrm{CdtB}$ is unaffected by this change, suggesting different intracellular pathways for the B subunit in the case of these two toxin types. It is noteworthy that Salmonella Typhi, which does not express either CdtA or CdtC subunits, uses a bacterial internalisation pathway to deliver the enzymatic CdtB subunit directly into the host cell (Haghjoo, 2004). These findings indicate that while the general model of CDT function may be correct, the key molecules that the subunit proteins interact with, and the pathways they take in the target cells may be different, and therefore should be investigated carefully in each CDT type.

Our study confirmed that in the case of E. coli CDT-V, as with other investigated CDT types (Lara-Tejero and Galán, 2001), both CdtA and CdtC are necessary for the toxin to be fully functional. However, as recent studies revealed considerable differences between the potential receptors of different CDT types, and between the potential roles of the subunit proteins, an important future task will be to determine the specific receptors responsible for the attachment of CDT-V to the target cell membrane, as well as the localisations of individual CDT-V subunits during the intoxication of target cells.

\section{Acknowledgement}

This work was supported by the Hungarian Scientific Research Fund (OTKA), grant number K 81252. 


\section{References}

Akifusa, S., Poole, S., Lewthwaite, J., Henderson, B. and Nair, S. P. (2001): Recombinant Actinobacillus actinomycetemcomitans cytolethal distending toxin proteins are required to interact to inhibit human cell cycle progression and to stimulate human leukocyte cytokine synthesis. Infect. Immun. 69, 5925-5930.

Belibasakis, G. N., Mattsson, A., Wang, Y., Chen, C. and Johansson, A. (2004): Cell cycle arrest of human gingival fibroblasts and periodontal ligament cells by Actinobacillus actinomycetemcomitans: involvement of the cytolethal distending toxin. APMIS 112, 674-685.

Bielaszewska, M., Sinha, B., Kuczius, T. and Karch, H. (2005): Cytolethal distending toxin from Shiga toxin-producing Escherichia coli $\mathrm{O} 157$ causes irreversible G2/M arrest, inhibition of proliferation, and death of human endothelial cells. Infect. Immun. 73, 552-562.

Comayras, C., Tasca, C., Pérès, S. Y., Ducommun, B., Oswald, E. and De Rycke, J. (1997): Escherichia coli cytolethal distending toxin blocks the HeLa cell cycle at the G2/M transition by preventing $\mathrm{Cdc} 2$ protein kinase dephosphorylation and activation. Infect. Immun. 65, 5088-5095.

Damek-Poprawa, M., Jang, J. Y., Volgina, A., Korostoff, J. and DiRienzo, J. M. (2012): Localization of Aggregatibacter actinomycetemcomitans cytolethal distending toxin subunits during intoxication of live cells. Infect. Immun. 80, 2761-2770.

De Rycke, J., Mazars, P., Nougayrede, J. P., Tasca, C., Boury, M., Herault, F., Valette, A. and Oswald, E. (1996): Mitotic block and delayed lethality in HeLa epithelial cells exposed to Escherichia coli BM2-1 producing cytotoxic necrotizing factor type 1. Infect. Immun. 64, 1694-1705.

Deng, K., Latimer, J. L., Lewis, D. A. and Hansen, E. J. (2001): Investigation of the interaction among the components of the cytolethal distending toxin of Haemophilus ducreyi. Biochem. Biophys. Res. Commun. 285, 609-615.

Elwell, C. A. and Dreyfus, L. A. (2000): DNAse I homologous residues in CdtB are critical for cytolethal distending toxin-mediated cell cycle arrest. Mol. Microbiol. 37, 952-963.

Eshraghi, A., Maldonado-Arocho, F. J., Gargi, A., Cardwell, M. M., Prouty, M. G., Blanke, S. R. and Bradley, K. A. (2010): Cytolethal distending toxin family members are differentially affected by alterations in host glycans and membrane cholesterol. J. Biol. Chem. 285, 18199-18207.

Gargi, A., Reno, M. and Blanke, S. R. (2012): Bacterial toxin modulation of the eukaryotic cell cycle: are all cytolethal distending toxins created equally? Front. Cell Infect. Microbiol. 2, 124.

Gargi, A., Tamilselvam, B., Powers, B., Prouty, M. G., Lincecum, T., Eshraghi, A., MaldonadoArocho, F. J., Wilson, B. A., Bradley, K. A. and Blanke, S. R. (2013): Cellular interactions of the cytolethal distending toxins from Escherichia coli and Haemophilus ducreyi. J. Biol. Chem. 288, 7492-7505.

Haghjoo, E. G. J. (2004): Salmonella Typhi encodes a functional cytolethal distending toxin that is delivered into host cells by a bacterial-internalization pathway. Proc. Natl Acad. Sci. USA 101, 4614-4619.

Hinenoya, A., Naigita, A., Ninomiya, K., Asakura, M., Shima, K., Seto, K., Tsukamoto, T., Ramamurthy, T., Faruque, S. M. and Yamasaki, S. (2009): Prevalence and characteristics of cytolethal distending toxin-producing Escherichia coli from children with diarrhea in Japan. Microbiol. Immunol. 53, 206-215.

Hinenoya, A., Shima, K., Asakura, M., Nishimura, K., Tsukamoto, T., Ooka, T., Hayashi, T., Ramamurthy, T., Faruque, S. M. and Yamasaki, S. (2014): Molecular characterization of cytolethal distending toxin gene-positive Escherichia coli from healthy cattle and swine in Nara, Japan. BMC Microbiol. 14, 97. 
Janka, A., Bielaszewska, M., Dobrindt, U., Greune, L., Schmidt, M. A. and Karch, H. (2003): Cytolethal distending toxin gene cluster in enterohemorrhagic Escherichia coli O157:H- and O157:H7: characterization and evolutionary considerations. Infect. Immun. 71, 3634-3638.

Lara-Tejero, M. and Galán, J. E. (2001): CdtA, CdtB, and CdtC form a tripartite complex that is required for cytolethal distending toxin activity. Infect. Immun. 69, 4358-4365.

McSweeney, L. A. and Dreyfus, L. A. (2005): Carbohydrate-binding specificity of the Escherichia coli cytolethal distending toxin CdtA-II and CdtC-II subunits. Infect. Immun. 73, 2051-2060.

Nesić, D., Hsu, Y. and Stebbins, C. E. (2004): Assembly and function of a bacterial genotoxin. Nature 429, 429-433.

Nougayrède, J., Taieb, F., De Rycke, J. and Oswald, E. (2005): Cyclomodulins: bacterial effectors that modulate the eukaryotic cell cycle. Trends Microbiol. 13, 103-110.

Orth, D., Grif, K., Dierich, M. P. and Würzner, R. (2006): Cytolethal distending toxins in Shiga toxin-producing Escherichia coli: alleles, serotype distribution and biological effects. J. Med. Microbiol. 55, 1487-1492.

Oswald, E., Nougayrède, J., Taieb, F. and Sugai, M. (2005): Bacterial toxins that modulate host cell-cycle progression. Curr. Opin. Microbiol. 8, 83-91.

Pérès, S. Y., Marchès, O., Daigle, F., Nougayrède, J. P., Herault, F., Tasca, C., De Rycke, J. and Oswald, E. (1997): A new cytolethal distending toxin (CDT) from Escherichia coli producing Cnf2 blocks HeLa cell division in G2/M phase. Mol. Microbiol. 24, 1095-1107.

Saiki, K., Konishi, K., Gomi, T., Nishihara, T. and Yoshikawa, M. (2001): Reconstitution and purification of cytolethal distending toxin of Actinobacillus actinomycetemcomitans. Microbiol. Immunol. 45, 497-506.

Sváb, D., Maróti, G., Horváth, B., Dobrindt, U. and Tóth, I. (2013): Sequence variability of P2-like prophage genomes carrying the cytolethal distending toxin V operon in Escherichia coli O157. Appl. Environ. Microbiol. 79, 4958-4964.

Taieb, F., Nougayrède, J., Watrin, C., Samba-Louaka, A. and Oswald, E. (2006): Escherichia coli cyclomodulin Cif induces G2 arrest of the host cell cycle without activation of the DNAdamage checkpoint-signalling pathway. Cell. Microbiol. 8, 1910-1921.

Tóth, I., Nougayrède, J., Dobrindt, U., Ledger, T. N., Boury, M., Morabito, S., Fujiwara, T., Sugai, M., Hacker, J. and Oswald, E. (2009a): Cytolethal distending toxin type I and type IV genes are framed with lambdoid prophage genes in extraintestinal pathogenic Escherichia coli. Infect. Immun. 77, 492-500.

Tóth, I., Schmidt, H., Kardos, G., Lancz, Z., Creuzburg, K., Damjanova, I., Pászti, J., Beutin, L. and Nagy, B. (2009b): Virulence genes and molecular typing of different groups of Escherichia coli $\mathrm{O} 157$ strains in cattle. Appl. Environ. Microbiol. 75, 6282-6291.

Yamada, T., Komoto, J., Saiki, K., Konishi, K. and Takusagawa, F. (2006): Variation of loop sequence alters stability of cytolethal distending toxin (CDT): crystal structure of CDT from Actinobacillus actinomycetemcomitans. Protein Sci. 15, 362-372. 